\title{
The chemistry of protoplanetary nebulae
}

\author{
P. M. Woods ${ }^{1,2}$, T. J. Millar ${ }^{2}$, E. Herbst ${ }^{3}$, and A. A. Zijlstra ${ }^{2}$ \\ ${ }^{1}$ European Southern Observatory, Alonso de Cordova 3107, Casilla 19001, Santiago 19, Chile \\ 2 Department of Physics, UMIST, PO Box 88, Manchester M60 1QD, UK \\ 3 Departments of Physics and Astronomy, The Ohio State University, Columbus, OH 43210, USA
}

Received 13 August 2002 / Accepted 13 February 2003

\begin{abstract}
We describe the chemistry occurring in a dense slab of outwardly expanding gas in the post-AGB phase of stellar evolution. Despite the high flux of UV photons from the central star, the intrinsic dust extinction in the slab prevents rapid photodissociation and allows chemical reactions to make a variety of gas-phase species, some complex. We find that chemical evolution ends when the intrinsic extinction falls below about 10 mag. At this point, rapid photodissociation occurs. As a particular example of our model, we discuss chemical synthesis in the protoplanetary nebula, CRL 618, and find that for many species we obtain good agreement between the model and observation, despite the complexity of the source.
\end{abstract}

Key words. molecular processes - stars: abundances - stars: AGB and post-AGB - stars: carbon - circumstellar matter stars: individual: CRL 618

\section{Introduction}

Stars in the late stages of stellar evolution have proven to be rich sources of molecules. Most molecules are detected in the highly opaque circumstellar envelopes (CSEs) of stars undergoing high mass loss on the Asymptotic Giant Branch (AGB). Carbon stars $([\mathrm{C}] /[\mathrm{O}]>1)$ show the richer chemistry, with over 50 different molecular species having been observed to date in the generic carbon star IRC+10216 (Olofsson 1997). Oxygen-rich stars, with $[\mathrm{C}] /[\mathrm{O}]<1$, show a more restricted chemistry. Most species detected in the envelope of IRC+10216 are long carbon chains; the largest molecule detected is $\mathrm{HC}_{9} \mathrm{~N}$ (e.g. see Cernicharo 2000; Millar 1988). After the end of the high-mass loss phase, AGB stars quickly evolve to high temperatures, leading to photodestruction of the molecules, and finally to ionisation and the formation of a planetary nebula $(\mathrm{PN})$.

The stage intermediate to the AGB and PN phases is called the post-AGB, or proto-planetary nebula (PPN), phase. Here, the presence of a hot star results in the emission of copious amounts of UV radiation which rapidly photodissociates and photoionises the remnant circumstellar envelope. The ages of PPNe are uncertain but are likely to be short, based on kinematic ages and the scarcity of such objects; typical estimates range from 200 to 1000 years for particular PPNe. However, Zijlstra et al. (2001) argue that the bipolar post-AGB stars show much longer time scales than indicated by the kinematic age,

Send offprint requests to: $\mathrm{P}$. M. Woods, e-mail: pwoods@eso.org and so the lifetime of some objects in the PPN phase may be longer than this.

Long carbon-chain molecules and cyanopolyynes have also been observed in post-AGB objects, and in particular the PPN, CRL 618. A recent series of papers by Cernicharo and collaborators (Cernicharo et al. 2001a; Cernicharo et al. 2001b; Herpin \& Cernicharo 2000) details detections of the carbonbased molecules $\mathrm{C}_{4} \mathrm{H}_{2}, \mathrm{C}_{6} \mathrm{H}_{2}, \mathrm{C}_{2} \mathrm{H}_{4}, \mathrm{CH}_{3} \mathrm{C}_{2} \mathrm{H}, \mathrm{CH}_{3} \mathrm{C}_{4} \mathrm{H}, \mathrm{C}_{6} \mathrm{H}_{6}$ (benzene) and the oxygen-bearing molecules $\mathrm{OH}$ and $\mathrm{H}_{2} \mathrm{O}$, using ISO. Benzene, the simplest aromatic molecule, and the radical $\mathrm{OH}$ have not been seen in IRC +10216 , although water has been detected, and is thought to arise from Kuiper Belt-like objects (Melnick et al. 2001).

The rich chemistry of CRL 618 is thought to be a result of the variety of physical conditions in the object. A central photon-dominated region (PDR) is irradiated by intense UV rays from the B0-type star (Martín-Pintado et al. 1993; Fong et al. 2001) and this is surrounded by the remnant AGB envelope and bipolar lobes with a high-velocity wind (Cernicharo et al. 1989). Detailed mapping has been carried out (e.g. Hajian et al. 1996; Neri et al. 1992) and the existence of a central torus of dust and gas seems clear (Lo \& Bechis 1976). The age of CRL 618 has been estimated to be between 200 years (Bujarrabal et al. 1988) and 600 years (Carsenty \& Solf 1982) using the expansion velocity of the bipolar lobes.

During the PPN phase, the visual extinction intrinsic to the circumstellar gas drops quickly as it expands while, at the same time, the star heats up. The emission of stellar UV photons then drives a quickly evolving photo-chemistry. In this paper, we present a time-dependent chemical model of this evolution 
and, as a particular example, compare the model with the abundances observed in CRL 618. Previous chemical models of the post-AGB phase have been described by Hasegawa et al. (2000) - for the young PN NGC 7027 - and by Howe et al. (1992) for PPNe. Hasegawa et al. (2000) used a PDR model to explain the molecular line observations of the neutral shell around NGC 7027, while Howe et al. (1992) used the interacting winds model to investigate the shock chemistry in the transition phase. The latter authors found that photodissociation by the central star was rapid and that, for molecules to survive to any extent, additional shielding, perhaps in the form of clumps, was needed. Howe et al. (1994) studied the chemistry in neutral globules within PNe and found reasonable agreement between their models and observations of the Helix Nebula, NGC 7293. One failing of their model was that the $\mathrm{HCO}^{+}$abundance was far below that observed. This is common to many models of the post-AGB phase (see also Ali et al. 2001) and is usually resolved by invoking increased ionisation, arising from enhanced $\mathrm{X}$-ray emission, for example.

\section{The physical model}

In order to follow the chemistry after the period of high massloss has stopped, we consider the remnant CSE to be in the form of a parcel, or slab, of gas moving away from the central star and assume that the material interior to the slab provides no extinction of the stellar UV flux. This assumption is reasonable given that dust formation does not occur after the AGB phase as well as the fact that the mass-loss rate is also smaller in the PPN phase. Thus, the shielding of molecules to the internal UV field comes from the slab material itself. Again, for simplicity, we have neglected the external interstellar UV field since it is much weaker than the stellar field and is itself extinguished by dust in the outer CSE.

If we consider CRL 618 as a representative of post-AGB objects, we note that the outwardly moving parcel we are modelling must co-exist with a fast bipolar outflow that probably has a wide opening angle, and which mass-loads material from the torus. Despite the large outward radial velocity of the outflow, the torus moves outward slowly. The chemistry we describe uses conditions appropriate for the torus; however, we ignore the interaction between the torus and the outflow. A recent study of such an interaction involving outflows in low-mass protostars (Viti et al. 2002) shows anomalously high abundances of $\mathrm{HCO}^{+}$which cannot be accounted for in our parcel model.

The gas density within the slab (or torus) is determined by the mass-loss rate, the expansion velocity and the initial radius at which we position the slab. The mass loss is assumed to occur only for that time necessary to eject the slab. We adopt a homogeneous slab of constant thickness, $\Delta r$, which, using mass conservation and the idea that the slab subtends a constant solid angle, implies that the internal density, $n(r)$, varies as $r^{-2}$, as does the intrinsic extinction, $A_{\mathrm{V}}(r)$, which is proportional to the product $n(r) \Delta r$. We use the thin slab approximation in the sense that we do not consider density inhomogeneities within the slab at any given time and we assume that all points in the slab experience the same extinction, which is taken to be the extinction through the entire slab. This is a simplification of the real situation, where the instantaneous conditions vary with radius and possibly with azimuthal angle. To convert model abundances of the selected parcel to true (observed) column densities would require a more complex model, e.g., by adopting a thick slab in which radial inhomogeneities play a role, or by integrating over a number of parcels at different distances from the star. Given the uncertainties in our knowledge of the geometrical structure of objects such as CRL618, we have chosen not to introduce these complexities. Comparison between our model and observations will assume that our thin-slab approach gives a satisfactory snap-shot description of the object.

Detailed calculations indicate that photodissociation is extremely rapid once $A_{\mathrm{V}}$ falls to below $10 \mathrm{mag}$ or so. This, and the fact that molecules are detected in some PPNe, implies that the expansion velocity of the slab must be low, or alternatively that the molecular gas must be in a long-lived disk. For example, for an expansion velocity of $15 \mathrm{~km} \mathrm{~s}^{-1}$, photodissociation destroys most molecules within 200 years. All calculations presented in this paper use an expansion velocity of $5 \mathrm{~km} \mathrm{~s}^{-1}$. This is the velocity attributed to the expanding torus in the Red Rectangle (Jura et al. 1995; Knapp et al. 2000), a slightly more evolved object, and close to that associated with the expanding torus of the peculiar star, HD 101584 (Olofsson \& Nyman 1999). Table 1 lists the initial conditions. For the UV flux, we adopt an initial enhancement factor $G / G_{0}=3.2 \times 10^{6}$ over the interstellar field, following Herpin \& Cernicharo (2000), who give an enhancement of $2 \times 10^{5}$ at $r=1 \times 10^{16} \mathrm{~cm}$. This enhancement factor becomes diluted with radius, as $1 / r^{2}$. We have also performed calculations for even larger enhancements but find little sensitivity to the flux - the result of efficient dust extinction when $A_{\mathrm{V}}$ is larger than about 20 mags. We use an enhanced interstellar radiation field rather than a stellar radiation field because tabulated photodissociation and photoionisation rates are based on the interstellar field. However, the temperature of a B-type star is not too dissimilar from the temperature of the interstellar radiation field, so that the extinction law used may introduce larger uncertainties than the choice of radiation field.

We have also considered enhanced ionisation by hard $\mathrm{X}$-rays. These can penetrate through a column density of $\approx 10^{23-24} \mathrm{~cm}^{-2}$ and increase the abundances of charged molecules (Glassgold et al. 1997), which are needed to enhance the speed of the chemistry. X-ray emission is a far more likely mechanism for the increased ionisation than enhanced cosmicray ionisation. Although both act in similar ways, the central source provides a possible source of X-rays; e.g. via accretion on a compact companion (Soker \& Kastner 2002) for which there is indirect evidence for several bipolar PPNe (Bujarrabal et al. 2001; Zijlstra et al. 2001). In contrast, the cosmic-ray intensity is mostly constant within the Galaxy, varying by no more than $30 \%$ within $15 \mathrm{kpc}$ Galactocentric distance (Strong \& Moskalenko 1989; Hunter et al. 1997). Because cosmic-ray ionisation is already a standard component of dark-cloud chemistry, we use this to simulate X-ray ionisation. Our standard model has an initial enhancement factor, $\zeta / \zeta_{0}=500$, which also is diluted as $1 / r^{2}$, until it reaches the standard interstellar value of $\zeta_{0}=1.3 \times 10^{-17} \mathrm{~s}^{-1}$. The effects of varying $\zeta$ are discussed in Sect. 4.6. To our knowledge CRL 618 has not been 
Table 1. Initial physical conditions.

\begin{tabular}{lc}
\hline \hline Parameter & Value \\
\hline $\mathrm{C} / \mathrm{O}$ ratio & 1.19 \\
$\dot{M}\left(M_{\odot} \mathrm{yr}^{-1}\right)$ & $3.2 \times 10^{-3}$ \\
$T(\mathrm{~K})$ & 250 \\
Inner radius, $r_{i}(\mathrm{~cm})$ & $2.5 \times 10^{15}$ \\
Expansion velocity, $v_{e}\left(\mathrm{~km} \mathrm{~s}^{-1}\right)$ & 5.0 \\
Initial $\mathrm{H}_{2}$ density, $n\left(\mathrm{H}_{2}\right)\left(\mathrm{cm}^{-3}\right)$ & $1.6 \times 10^{9}$ \\
Initial extinction, $A_{\mathrm{V}}(\mathrm{mags})$ & 160 \\
Slab thickness, $\Delta r(\mathrm{~cm})$ & $9.4 \times 10^{13}$ \\
Initial UV flux enhancement, $G / G_{0}$ & $3.2 \times 10^{6}$ \\
Initial CR enhancement, $\zeta / \zeta_{0}$ & 500 \\
\hline
\end{tabular}

detected in X-ray emission (Guerrero et al. 2000), but it must be pointed out that soft X-rays are likely to be rapidly absorbed in material of column density $>10^{21} \mathrm{~cm}^{-2}$.

The model assumes solar abundances with the exception of the enhanced carbon abundance. The $\mathrm{C} / \mathrm{O}$ ratio (by number) is taken as 1.19. This is higher than average for Galactic carbon stars (1.05-1.1: Matsuura et al. 2002) but is the value found for the high mass-loss star IRC+10216 (Millar et al. 2001). The temperature of the moving slab is assumed to remain constant at $250 \mathrm{~K}$ despite the weakening of the stellar radiation field and lowering of the ionisation rate with time. Although this simplifying assumption can be removed, most of the important chemical reactions in our network do not evince a strong temperature dependence. More detail on the effects of temperature variation is given in Sect. 5.2.

\section{The chemical model}

Since we wish to follow the chemistry of a carbon-rich postAGB object, our reaction network is based on that which Millar et al. (2000) used to describe the chemistry in the CSE around IRC +10216 . This network was primarily developed to describe the formation of large carbon chain molecules, including the cyanopolyynes, with up to 23 carbon atoms. The chemistry incorporates an extensive radical chemistry, especially involving $\mathrm{C}_{2}$ and $\mathrm{C}_{2} \mathrm{H}$, the daughter products of $\mathrm{C}_{2} \mathrm{H}_{2}$, which are far more abundant in circumstellar envelopes than in dense interstellar clouds. We also include the rapid neutral-neutral reactions discussed in Millar \& Herbst (1994). The network also contains reactions involving negative ions, which turn out to be important in IRC +10216 but are less significant here due to the lower fractional ionisation in the higher density slab adopted in the PPN model. The species $\mathrm{H}_{2} \mathrm{O}$ and $\mathrm{OH}$, which have been detected in CRL 618, are not included in the reaction network. These molecules are thought to be formed in shocks or vaporization in carbon-rich circumstellar environments. In summary, our model comprises some 407 species in 6 elements $(\mathrm{H}, \mathrm{He}$, $\mathrm{C}, \mathrm{N}, \mathrm{O}, \mathrm{S}$ ) with 3880 reactions. Initial fractional abundances of parent molecules relative to $\mathrm{H}_{2}$ are given in Table 2. These are "standard" initial abundances, in the sense that these were used for the previous model of IRC +10216 , and are typical of a
Table 2. Adopted initial fractional abundances of parent species with respect to $n\left(\mathrm{H}_{2}\right)$.

\begin{tabular}{lc}
\hline \hline Species & Initial fractional abundance \\
\hline $\mathrm{He}$ & $1.5 \times 10^{-1}$ \\
$\mathrm{CO}$ & $6.0 \times 10^{-4}$ \\
$\mathrm{~N}_{2}$ & $2.0 \times 10^{-4}$ \\
$\mathrm{C}_{2} \mathrm{H}_{2}$ & $5.0 \times 10^{-5}$ \\
$\mathrm{HCN}$ & $8.0 \times 10^{-6}$ \\
$\mathrm{CS}$ & $4.0 \times 10^{-6}$ \\
$\mathrm{CH}$ & $2.0 \times 10^{-6}$ \\
$\mathrm{NH}_{3}$ & $2.0 \times 10^{-6}$ \\
$\mathrm{H}_{2} \mathrm{~S}$ & $1.0 \times 10^{-6}$ \\
\hline
\end{tabular}

carbon-rich AGB star. We take the final abundances of the AGB phase as initial abundances for the PPN phase. Self-shielding of CO is included using the approach of Mamon et al. (1988). Self-shielding of somewhat less abundant parent species is not considered, although it may play a role.

\section{Results}

Figure 1 shows the fractional abundances of several molecules relative to $n\left(\mathrm{H}_{2}\right)$ as a function of radial distance from the central star. The abundances all show one of two behaviours. In the case of parent molecules, a decrease at about $9 \times 10^{15} \mathrm{~cm}$ occurs when photodissociation finally cuts in. The rapid decrease in abundances is due to the fact that the radiation field is much enhanced over the interstellar field, so that the photodissociation time scales are very short at small $A_{\mathrm{V}}$. For daughter products, there is a rise in abundance as parents are photodissociated but again they experience a very rapid fall-off once photons dominate their destruction.

Although the fractional abundances of daughter products tend to be highest in the photodissociation region, the picture is different if one considers column densities since the overall gas density decreases with increasing radius as $r^{-2}$. In terms of column densities, those of daughter products tend to be much flatter vs. radius while those of parent species decrease strongly even before the photodissociation region. In general, the picture that emerges is that ion-molecule chemistry occurs at the smallest radii considered due to the enhanced ionisation rate. The enhanced ionisation rate also results in large numbers of photons induced by cosmic ray bombardment; these photons produce radicals and ions and accelerate the chemistry. The overall chemistry at small radii produces small fractional abundances of many daughter species but the column densities are enhanced by the high overall initial density. The production of large column densities happens very quickly because of the small time interval between collisions at small radii $(1.0 \mathrm{~s})$.

Molecule formation, and especially complex molecule formation, is unexpectedly efficient in the photodissociation region. For example, the cyanopolyynes up to $\mathrm{HC}_{11} \mathrm{~N}$ all have fractional abundances above $10^{-7}$ once the slab has reached $\sim 9 \times 10^{15} \mathrm{~cm}$, while benzene and its derivative, 


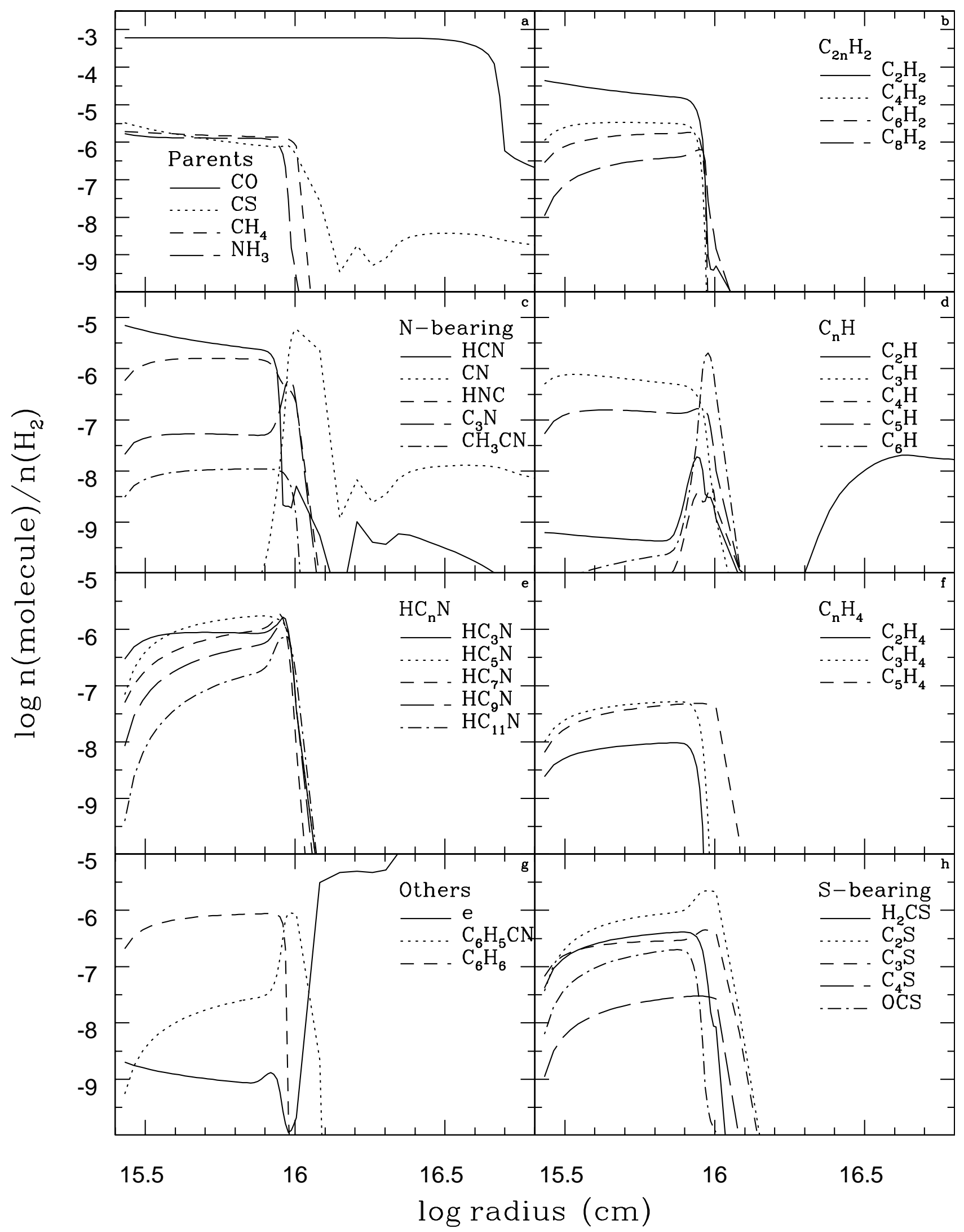

Fig. 1. The variation of molecular abundances with radial distance in the standard PPN model, for an initial ionisation rate 500 times that of the standard interstellar value.

benzonitrile, $\mathrm{C}_{6} \mathrm{H}_{5} \mathrm{CN}$, reach a maximum fractional abundance of about $\sim 10^{-6}$. We discuss the reactions contributing to the formation of these molecules in the following sub-sections, emphasizing the dominant processes at a radius of $8.9 \times 10^{15} \mathrm{~cm}$, 
which is reached in $\sim 425$ years. This radius is approximately where the majority of species are most abundant.

\subsection{Small molecules}

In this section we discuss the formation routes to simple molecules such as $\mathrm{HNC}, \mathrm{CN}$ and $\mathrm{C}_{2} \mathrm{H}$ and those containing sulphur.

\subsubsection{HNC}

$\mathrm{HNC}$ is made in the dissociative recombination of $\mathrm{HCNH}^{+}$, which itself is formed by proton transfer reactions with the parent molecule, HCN. The column density of HNC is less than that of $\mathrm{HCN}$ until around $8.9 \times 10^{15} \mathrm{~cm}$, when loss of $\mathrm{HCN}$ in reactions with $\mathrm{C}_{2 n} \mathrm{H}(n \geq 2)$ becomes significant. HNC is also lost through analogous reactions, mostly with $\mathrm{C}_{2} \mathrm{H}$. The time at which the abundances reach equality is roughly 425 years. Herpin \& Cernicharo (2000) found equal column densities of HCN and HNC in CRL 618. This equality is in contrast to the case of IRC+10216, in which HCN is much more abundant than HNC. The difference is unlikely to reflect only the age of the shell: IRC +10216 has a large shell which indicates extended mass loss. But there are other differences: (i) IRC+10216 is an AGB star with continuing mass loss, while CRL 618 is a post-AGB star with a detached shell. The observed column densities in IRC +10216 are affected by LTE chemistry in the photosphere; (ii) IRC +10216 has a higher expansion velocity than the value assumed here for the torus of CRL 618 which reduces the timescale available for the chemistry (CRL 618 also has a very fast polar outflows which we do not model, but the dominant molecular mass is likely to be in the shielded torus); (iii) the shell density in the wind of IRC +10216 is likely lower than in the disk of CRL 618, due to the (expected) difference in expansion velocities, which results in a slower conversion of parent $\mathrm{HCN}$ to HNC.

The increasing HNC/HNC abundance ratio might be a useful indicator of evolution in the PPN phase. The difference with IRC +10216 suggests the possibility that it can be used to measure the product of density and expansion timescale.

\subsection{2. $\mathrm{CN}$}

$\mathrm{CN}$ is the daughter product of the parent molecule HCN. Its radial abundance distribution is rather sharply peaked at around $1-3 \times 10^{16} \mathrm{~cm}$, because it is formed by the photodissociation of $\mathrm{HCN}$ and $\mathrm{HNC}$. Its abundance inside this radius is much less than that of $\mathrm{HNC}$, also formed from $\mathrm{HCN}$, because of the rapid neutral reactions it undergoes with the hydrocarbons of the type $\mathrm{C}_{2 n} \mathrm{H}_{2}$, to form the cyanopolyyne chain.

\subsection{3. $\mathrm{C}_{2} \mathrm{H}$}

The ethynyl radical is produced from parent $\mathrm{C}_{2} \mathrm{H}_{2}$ by $\mathrm{UV}$ and $\mathrm{CR}$-induced photons. It is a very reactive radical and is involved in the creation of many other chain species. It reacts with $\mathrm{S}$ and
CS to form $\mathrm{C}_{2} \mathrm{~S}$ and $\mathrm{C}_{3} \mathrm{~S}$, with $\mathrm{C}_{2} \mathrm{H}_{2}$ to form $\mathrm{C}_{4} \mathrm{H}_{2}$, and helps build larger chains by insertion reactions (see below).

\subsubsection{OCS}

OCS if formed in a series of reactions initiated by the reaction:

$\mathrm{S}+\mathrm{CH}_{2} \longrightarrow \mathrm{HCS}+\mathrm{H}$

followed primarily by :

$\mathrm{O}+\mathrm{HCS} \longrightarrow \mathrm{OCS}+\mathrm{H}$

and by the radiative association reaction:

$\mathrm{S}+\mathrm{CO} \longrightarrow \mathrm{OCS}+\mathrm{h} v$.

It has a fractional abundance of $\sim 2 \times 10^{-8}$ around $8.9 \times$ $10^{15} \mathrm{~cm}$.

\subsection{5. $\mathrm{H}_{2} \mathrm{CS}$}

Thioformaldehyde is formed primarily in the neutral reaction:

$\mathrm{S}+\mathrm{CH}_{3} \longrightarrow \mathrm{H}_{2} \mathrm{CS}+\mathrm{H}$,

and destroyed in proton transfer reactions, and by UV and CRinduced UV photons. Its formation is very efficient and its fractional abundance is $2.7 \times 10^{-7}$ at $8.9 \times 10^{15} \mathrm{~cm}$.

\subsection{Hydrocarbons}

The formation of hydrocarbons is initiated by reactions involving the parent molecule $\mathrm{C}_{2} \mathrm{H}_{2}$ (acetylene) and its daughters ${ }^{1}$ $\mathrm{C}_{2} \mathrm{H}$ and $\mathrm{C}_{2} \mathrm{H}_{2}^{+}$(see reactions (1) to (4)). These reactions can be generalised to synthesise larger, even-numbered hydrocarbons $\left(\mathrm{C}_{2 n} \mathrm{H}_{m}\right)$ (reactions (5) and (6)).

$$
\begin{array}{ll}
\mathrm{C}_{2} \mathrm{H}+\mathrm{C}_{2} \mathrm{H}_{2} & \longrightarrow \mathrm{C}_{4} \mathrm{H}_{2}+\mathrm{H} \\
\mathrm{C}_{2} \mathrm{H}+\mathrm{C}_{2} \mathrm{H} & \longrightarrow \mathrm{C}_{4} \mathrm{H}+\mathrm{H} \\
\mathrm{C}_{2} \mathrm{H}_{2}^{+}+\mathrm{C}_{2} \mathrm{H}_{2} & \longrightarrow \mathrm{C}_{4} \mathrm{H}_{3}^{+}+\mathrm{H} \\
\mathrm{C}_{2} \mathrm{H}_{2}^{+}+\mathrm{C}_{2} \mathrm{H}_{2} & \longrightarrow \mathrm{C}_{4} \mathrm{H}_{2}^{+}+\mathrm{H}_{2} \\
\mathrm{C}_{2} \mathrm{H}+\mathrm{C}_{2 n} \mathrm{H}_{2} & \longrightarrow \mathrm{C}_{2 n+2} \mathrm{H}_{2}+\mathrm{H} \\
\mathrm{C}_{2} \mathrm{H}_{2}^{+}+\mathrm{C}_{2 n} \mathrm{H}_{m} \longrightarrow \mathrm{C}_{2 n+2} \mathrm{H}_{m+1}^{+}+\mathrm{H} .
\end{array}
$$

Because these reactions involve the abundant parent acetylene, the abundances of the even-numbered hydrocarbons $\left(\mathrm{C}_{2 n} \mathrm{H}_{2}\right)$ are generally larger than those of the odd-numbered hydrocarbons $\left(\mathrm{C}_{2 n+1} \mathrm{H}_{2}\right)$. Reactions of atomic carbon (which initially comes from CR-induced photodissociation of $\mathrm{CO}$ ) with $\mathrm{C}_{2} \mathrm{H}_{2}$ and $\mathrm{C}_{2} \mathrm{H}$, and other even-numbered hydrocarbons, also help increase the chain length and produce the odd-numbered species $\mathrm{C}_{2 n+1}$ and $\mathrm{C}_{2 n+1} \mathrm{H}$. Figure 1 shows that the odd-numbered hydrocarbon radicals, $\mathrm{C}_{2 n+1} \mathrm{H}$, are more abundant than their even-numbered counterparts for distances less than about $8 \times$ $10^{15} \mathrm{~cm}$. This is primarily due to two reasons: (i) the $\mathrm{C}_{2 n} \mathrm{H}$ species are destroyed more rapidly in reactions with parent $\mathrm{HCN}$ and $\mathrm{C}_{2} \mathrm{H}_{2}$, and (ii) their formation rates, which depend

\footnotetext{
${ }^{1} \mathrm{C}_{2} \mathrm{H}_{2}+\mathrm{h} v \longrightarrow \mathrm{C}_{2} \mathrm{H}+\mathrm{H} ; \quad \mathrm{C}_{2} \mathrm{H}_{2}+\mathrm{h} v \longrightarrow \mathrm{C}_{2} \mathrm{H}_{2}^{+}+e$
} 
ultimately on the CR-induced photodissociation of $\mathrm{C}_{2} \mathrm{H}_{2}$, are slower than those of the odd-numbered chain, which is driven by the $\mathrm{C}+\mathrm{C}_{2} \mathrm{H}_{2}$ reaction.

Methane, $\mathrm{CH}_{4}$, is another parent molecule involved in hydrocarbon formation. Typically it is about ten times less abundant than acetylene in C-rich CSEs. Synthesis of larger hydrocarbons proceeds at large radii via $\mathrm{C}^{+}$insertion reactions or condensation reactions, e.g.,

$$
\begin{aligned}
& \mathrm{CH}_{4}+\mathrm{C}^{+} \longrightarrow \mathrm{C}_{2} \mathrm{H}_{2}^{+}+\mathrm{H}_{2} \\
& \mathrm{CH}_{4}+\mathrm{C}^{+} \longrightarrow \mathrm{C}_{2} \mathrm{H}_{3}^{+}+\mathrm{H} \\
& \mathrm{CH}_{4}+\mathrm{CH}_{3}^{+} \longrightarrow \mathrm{C}_{2} \mathrm{H}_{5}^{+}+\mathrm{H}_{2} .
\end{aligned}
$$

Chain-lengthening then proceeds via further condensation reactions with $\mathrm{C}_{2} \mathrm{H}_{2}$ and $\mathrm{C}_{2} \mathrm{H}$, plus reactions with $\mathrm{C}$ and $\mathrm{C}^{+}$.

\subsection{Cyanopolyynes}

The cyanopolyynes are synthesised via a variety of mechanisms. Let us first consider the simplest member of the series $\mathrm{HC}_{3} \mathrm{~N}$. One important reaction to produce this species uses $\mathrm{CN}$ and acetylene (Howe \& Millar 1990):

$\mathrm{CN}+\mathrm{C}_{2} \mathrm{H}_{2} \longrightarrow \mathrm{HC}_{3} \mathrm{~N}+\mathrm{H}$.

This reaction is the dominant source of $\mathrm{HC}_{3} \mathrm{~N}$ in interstellar clouds. Here it also dominates throughout most of the slab. Another important reaction is the (relatively slow) process between $\mathrm{HCN}$ and $\mathrm{C}_{2} \mathrm{H}$ :

$\mathrm{HCN}+\mathrm{C}_{2} \mathrm{H} \longrightarrow \mathrm{HC}_{3} \mathrm{~N}+\mathrm{H}$.

This reaction has now been measured (Hoobler \& Leone 1997) and calculated by Fukuzawa \& Osamura (1997) to have a small activation energy barrier. This barrier effectively prevents reaction (8) at the chosen temperature of $250 \mathrm{~K}$. A third important reaction is the analogous process involving $\mathrm{HNC}$ :

$\mathrm{HNC}+\mathrm{C}_{2} \mathrm{H} \longrightarrow \mathrm{HC}_{3} \mathrm{~N}+\mathrm{H}$,

which actually becomes the dominant production method of $\mathrm{HC}_{3} \mathrm{~N}$ just before photons become appreciable. After photons become appreciable, $\mathrm{HC}_{3} \mathrm{~N}$ is produced mainly by the reaction between atomic nitrogen and $\mathrm{C}_{3} \mathrm{H}_{2}$.

There are two main routes to the production of more complex cyanopolyynes in our model. First, reactions between smaller cyanopolyynes and hydrocarbon radicals serve to produce larger cyanopolyynes; viz.,

$\mathrm{HC}_{2 n+1} \mathrm{~N}+\mathrm{C}_{2} \mathrm{H} \longrightarrow \mathrm{HC}_{2 n+3} \mathrm{~N}+\mathrm{H}, \quad n \geq 1$,

$\mathrm{HC}_{2 n-3} \mathrm{~N}+\mathrm{C}_{4} \mathrm{H} \longrightarrow \mathrm{HC}_{2 n+1} \mathrm{~N}+\mathrm{H}, \quad n \geq 2$,

by adding carbon atoms to the skeletal chain of the cyanopolyynes. Secondly, $\mathrm{CN}$ and $\mathrm{HCN}$ react with evennumbered hydrocarbons and radicals to produce the larger cyanopolyynes directly:

$$
\begin{aligned}
& \mathrm{CN}+\mathrm{C}_{2 n} \mathrm{H}_{2} \longrightarrow \mathrm{HC}_{2 n+1} \mathrm{~N}+\mathrm{H} \\
& \mathrm{HCN}+\mathrm{C}_{2 n} \mathrm{H} \longrightarrow \mathrm{HC}_{2 n+1} \mathrm{~N}+\mathrm{H} .
\end{aligned}
$$

Figure 1 shows that cyanopolyyne synthesis is very efficient under the conditions of our model, as it was found to be in IRC+10216, in agreement with the observations. The cyanopolyynes up to $\mathrm{HC}_{9} \mathrm{~N}$ have been detected in $\mathrm{IRC}+10216$; this is also the largest cyanopolyyne detected to date in a postAGB object, in this case the PPN CRL 2688 (Truong-Bach et al. 1993). The cyanopolyyne $\mathrm{HC}_{9} \mathrm{~N}$ is formed in our model at a distance of $8 \times 10^{15} \mathrm{~cm}$ by the following reactions: $\mathrm{C}_{2} \mathrm{H}+$ $\mathrm{HC}_{7} \mathrm{~N}(58 \%) ; \mathrm{C}_{4} \mathrm{H}+\mathrm{HC}_{5} \mathrm{~N}(17 \%) ; \mathrm{C}_{8} \mathrm{H}+\mathrm{HCN}(16 \%)$. Loss at this radius is mostly through dissociative recombination (18\%), and reactions which build the larger cyanopolyynes (68\%).

\subsection{Organo-sulphur chains}

The organo-sulphur chains, $\mathrm{C}_{2} \mathrm{~S}, \mathrm{C}_{3} \mathrm{~S}$ and $\mathrm{C}_{4} \mathrm{~S}$ are included in this model. The major reactions in their formation involve atomic sulphur and parent $\mathrm{CS}$ :

$$
\begin{aligned}
\mathrm{S}+\mathrm{C}_{2} \mathrm{H} & \longrightarrow \mathrm{C}_{2} \mathrm{~S}+\mathrm{H} \\
\mathrm{CS}+\mathrm{C}_{2} \mathrm{H} & \longrightarrow \mathrm{C}_{3} \mathrm{~S}+\mathrm{H} \\
\mathrm{S}+\mathrm{C}_{4} \mathrm{H}_{3}^{+} & \longrightarrow \mathrm{HC}_{4} \mathrm{~S}^{+}+\mathrm{H}_{2} \\
\mathrm{~S}+\mathrm{C}_{4} \mathrm{H}_{2}^{+} & \longrightarrow \mathrm{HC}_{4} \mathrm{~S}^{+}+\mathrm{H} \\
\mathrm{HC}_{4} \mathrm{~S}^{+}+\mathrm{e} & \longrightarrow \mathrm{C}_{2} \mathrm{~S}+\mathrm{C}_{2} \mathrm{H} \\
& \longrightarrow \mathrm{C}_{3} \mathrm{~S}+\mathrm{CH} \\
& \longrightarrow \mathrm{C}_{4} \mathrm{~S}+\mathrm{H} .
\end{aligned}
$$

The analogous reactions to form larger organo-sulphur chains have not been included in this model; Millar et al. (2001) have discussed this possibility for the case of IRC +10216 . Our calculated abundances for $\mathrm{C}_{2} \mathrm{~S}$ and $\mathrm{C}_{3} \mathrm{~S}$ are significant, that for $\mathrm{C}_{4} \mathrm{~S}$ less so as it is formed in an ion-molecule, rather than neutral-neutral, reaction. Millar et al. (2001) have shown that the spin-allowed reaction:

$\mathrm{S}+\mathrm{C}_{4} \mathrm{H} \longrightarrow \mathrm{C}_{4} \mathrm{~S}+\mathrm{H}$

increases the abundance of $\mathrm{C}_{4} \mathrm{~S}$ in $\mathrm{IRC}+10216$ by over an order of magnitude.

\subsection{Benzene}

A mechanism for the formation of benzene, $\mathrm{C}_{6} \mathrm{H}_{6}$, in the interstellar medium has been discussed by McEwan et al. (1999), based on their laboratory studies of the reactions of $\mathrm{H}$ and $\mathrm{H}_{2}$ with hydrocarbon ions. For the conditions adopted here we find a more efficient synthesis, with fractional abundances of $\sim 10^{-6}$ being reached. At a distance of $\sim 8 \times 10^{15} \mathrm{~cm}$, the major route to the production of benzene is:

$$
\begin{aligned}
& \mathrm{H}_{3}^{+}+\mathrm{CO} \longrightarrow \mathrm{HCO}^{+}+\mathrm{H}_{2} \\
& \mathrm{~N}_{2} \mathrm{H}^{+}+\mathrm{CO} \longrightarrow \mathrm{HCO}^{+}+\mathrm{N}_{2} \\
& \mathrm{HCO}^{+}+\mathrm{C}_{4} \mathrm{H}_{2} \longrightarrow \mathrm{C}_{4} \mathrm{H}_{3}^{+}+\mathrm{CO} \\
& \mathrm{C}_{2} \mathrm{H}_{2}^{+}+\mathrm{C}_{2} \mathrm{H}_{2} \longrightarrow \mathrm{C}_{4} \mathrm{H}_{3}^{+}+\mathrm{H} \\
& \mathrm{C}_{2} \mathrm{H}_{3}^{+}+\mathrm{C}_{2} \mathrm{H}_{2} \longrightarrow \mathrm{C}_{4} \mathrm{H}_{3}^{+}+\mathrm{H}_{2} \\
& \mathrm{C}_{4} \mathrm{H}_{3}^{+}+\mathrm{C}_{2} \mathrm{H}_{2} \longrightarrow c-\mathrm{C}_{6} \mathrm{H}_{5}^{+}+\mathrm{h} v \\
& c-\mathrm{C}_{6} \mathrm{H}_{5}^{+}+\mathrm{H}_{2} \longrightarrow c-\mathrm{C}_{6} \mathrm{H}_{7}^{+}+\mathrm{h} v \\
& c-\mathrm{C}_{6} \mathrm{H}_{7}^{+}+\mathrm{e} \longrightarrow c-\mathrm{C}_{6} \mathrm{H}_{6}+\mathrm{H}
\end{aligned}
$$

where the ionised acetylene (Eq. (13)), which starts off the chain, is produced by cosmic-ray induced photoionisation of 


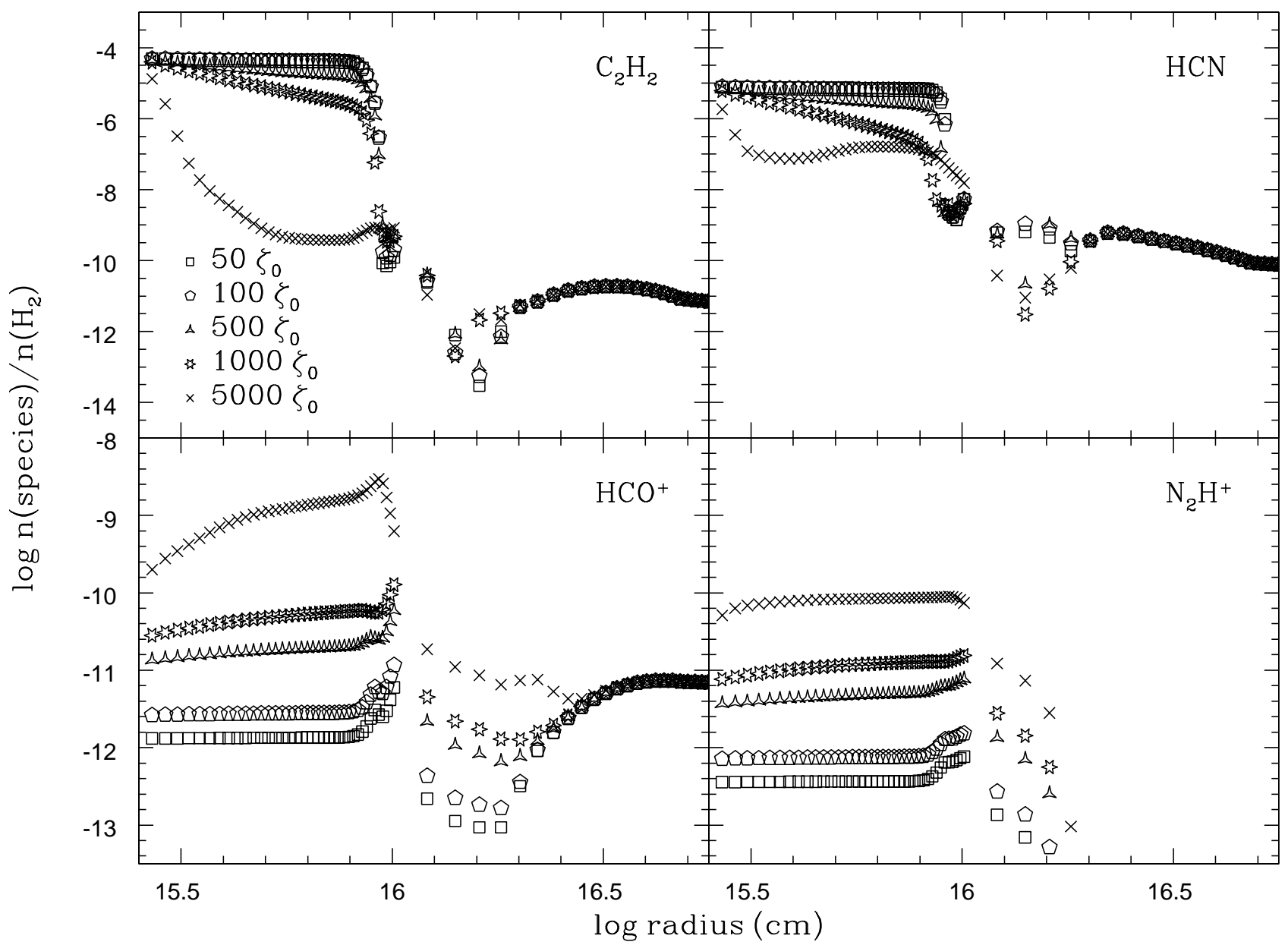

Fig. 2. The influence of the ionisation rate on the abundances of some species is shown. Once the initial rate becomes more than about 500 times larger than the standard interstellar rate, parent molecules get destroyed rapidly.

acetylene, and $\mathrm{C}_{2} \mathrm{H}_{3}^{+}$is formed from proton transfer reactions with acetylene.

The formation of $\mathrm{C}_{2} \mathrm{H}_{3}^{+}$(Eq. (14)) at small radii is highly dependent on abundances of $\mathrm{HCO}^{+}$and $\mathrm{H}_{3}^{+}$, which in turn are dependent on the flux of ionising radiation. The synthesis of benzene is examined in more detail in Woods et al. (2002).

At this radius, benzene is destroyed mainly through reaction with $\mathrm{CN}$, to form benzonitrile, $\mathrm{C}_{6} \mathrm{H}_{5} \mathrm{CN}$, via the reaction:

$\mathrm{CN}+\mathrm{C}_{6} \mathrm{H}_{6} \longrightarrow \mathrm{C}_{6} \mathrm{H}_{5} \mathrm{CN}+\mathrm{H}$

and by proton transfer reactions followed by dissociative recombination.

\subsection{Ionisation}

In these models we have adopted an initial ionisation rate some 500 times larger than the standard interstellar rate of $1.3 \times$ $10^{-17} \mathrm{~s}^{-1}$ in order to simulate the larger ionisation that may be caused by X-ray emission from the central object. In order to test the sensitivity of our results to this rate, we have run a series of models with the rate enhanced over the interstellar value by factors of 10-5000. Figure 2 shows the results of some of these calculations for a small number of selected species. For initial ionisation enhancement factors greater than 500, we find that the ionisation gets so large that parent molecules, such as $\mathrm{C}_{2} \mathrm{H}_{2}$ and $\mathrm{HCN}$, are destroyed very rapidly. The figure shows, for example, that the abundance of $\mathrm{C}_{2} \mathrm{H}_{2}$ is reduced by over 5 orders of magnitude by the time the slab has reached about $1 \times 10^{16} \mathrm{~cm}$ (or within a time-scale of 500 years for an expansion velocity of $5 \mathrm{~km} \mathrm{~s}^{-1}$ ) with an initial ionisation enhancement of 5000 . Thus, we neglect enhancements larger than 500 as producing molecular slabs which are too short lived to be of interest. With our adopted ionisation rate, the precipitous drop in abundance of parent species occurs mainly in the photodissociation region at a radius of $1 \times 10^{16} \mathrm{~cm}$.

\section{The proto-planetary nebula, CRL 618}

We apply our model of PPN chemistry to the specific case of CRL 618. CRL 618 is a particularly well-observed PPN, with a large amount of molecular data available.

\subsection{Comparison with $\mathrm{CO}$ observations}

Observations of CO have been able to give structural, kinematic and mass-loss information. Various papers (e.g. Herpin \& Cernicharo 2000; Meixner et al. 1998; Hajian et al. 1996; Martín-Pintado et al. 1995; Phillips et al. 1992; Neri et al. 1992) 


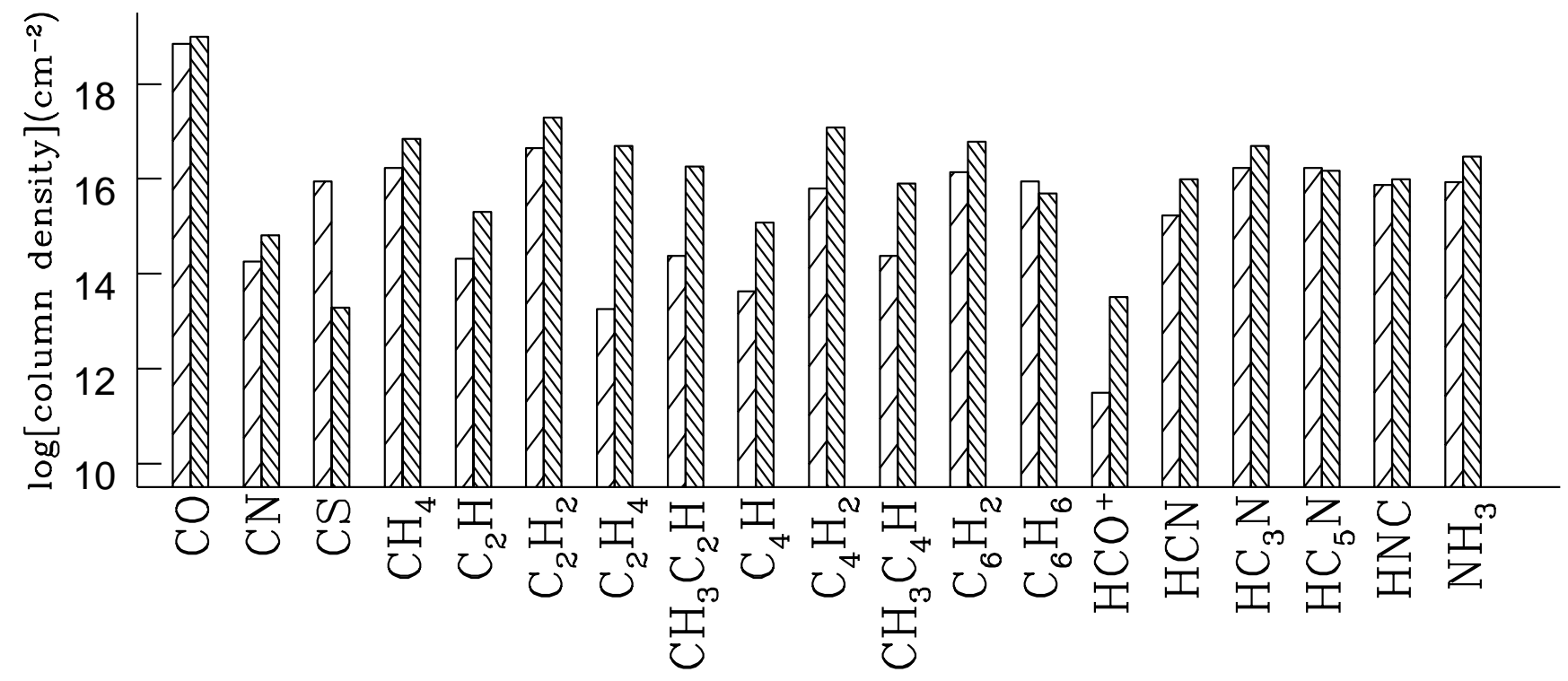

Fig. 3. Comparison between observed (heavy hashing) and model (light hashing) column densities at $8.9 \times 10^{15} \mathrm{~cm}$, for CRL 618 .

have reported quantitative sizes for structures within CRL618. Reasonable distance estimates range between $1300 \mathrm{pc}$ (e.g. Sopka et al. 1989) and $1700 \mathrm{pc}$ (e.g. Bujarrabal et al. 1988), so for simplicity we will assume that CRL 618 is $1500 \mathrm{pc}$ away. The "core" is given as 15" (Hajian et al.) or 19" (Meixner et al.) which corresponds to radial distances from the star of 1.7 and $2.1 \times 10^{17} \mathrm{~cm}$ at $1500 \mathrm{pc}$. At $19 \mathrm{~km} \mathrm{~s}^{-1}$, the envelope expansion velocity attributed to CRL 618 by Fuente et al. (1998), among others, this gives an expansion timescale of some 30003500 years. Also, Hajian et al. identify a region of high density subtending $\approx 3-4$ ", which equates to a radial distance of $3.4-4.5 \times 10^{16} \mathrm{~cm}$ at $1500 \mathrm{pc}$. Martín-Pintado et al. observed a dense disk, of some $1^{\prime \prime}$ in extent, which surrounds a molecular "hole" of 0.' 42, which could be the edge of the Hir region. In our model of a circumstellar slab, CO is predicted to be reasonably abundant out until around $5 \times 10^{16} \mathrm{~cm}\left(\sim 44^{\prime \prime} 5\right.$ on the sky), although it still has abundances of $\approx 10^{-7}$ at larger distances. Observable species (other than $\mathrm{CO}$ ) are depleted by a radial distance of $0.9-1.3 \times 10^{16} \mathrm{~cm}$, which is $0 .{ }^{\prime} 8-1^{\prime \prime}$ on the sky. The region modelled in the paper, a retarded disk with very slow expansion, would correspond to the dense region found by Martín-Pintado et al. (1995) in $\mathrm{NH}_{3}$ emission.

\subsection{Comparison with other molecular observations}

To compare our calculated column densities with observed values, we choose a radial distance $-8.9 \times 10^{15} \mathrm{~cm}-$ close to the distance where photodissociation destroys the molecules heavily. This radial distance has some observational significance, as discussed previously. At smaller distances, some daughter species have not yet achieved large abundances, although other daughters can have peaked, whilst parents are most abundant at the inner radius. At distances closer to the radius where photodestruction sets in $\left(0.9-1.3 \times 10^{16} \mathrm{~cm}\right)$, other daughter species achieve even larger column densities than at the distance chosen. Table 3 gives our calculated column densities at the chosen distance of $8.9 \times 10^{15} \mathrm{~cm}$, along with their peak values, and compares both of these to observations where available. The observational results shown in Table 3 are only those reported as column densities; results reported as fractional abundances are discussed separately below, and shown in Table 4. The purpose of including the peak abundances is to show that if the model does not produce sufficient material at the radial distance chosen, it in all likelihood can do so at other radial distances. At the distance chosen, the calculated abundances are changing rapidly (or are about to do so at slightly larger distances), so that a truly quantitative comparison between theory and observation is difficult. In addition, it is clear that there are widely divergent values for the observed column densities of some species, notably $\mathrm{HCN}, \mathrm{HNC}, \mathrm{HC}_{3} \mathrm{~N}$, and $\mathrm{HC}_{5} \mathrm{~N}$, and that in particular the column densities derived from single-dish radio telescopes are usually much lower than those derived from the ISO observations. This is probably a result of both beam dilution and the fact that the millimetre-wave observations are not as sensitive to the hot dense gas as the infrared observations. A further possibility results from the complexity of the source, with the result that pointing errors could also be significant. Consider the millimetre-wave results of Bujarrabal et al. (1988, 1994), who give fractional abundances of several molecules also observed by ISO. Even within these two papers, differences in abundances of an order of magnitude can occur (for CS, $\mathrm{HCN}, \mathrm{HC}_{3} \mathrm{~N}$ ). In Bujarrabal et al. (1994), the fractional abundance of $\mathrm{CS}$ is given as $4.1 \times 10^{-7}$, equivalent to a column density of $4.9 \times 10^{15} \mathrm{~cm}^{-2}$, compared to $1.9 \times 10^{13} \mathrm{~cm}^{-2}$ derived by Bachiller et al. (1997), also from millimetre-wave observations. At this point, we simply say that our abundances give a reasonable, but not perfect, match to the observed values. Certainly, and most importantly, the degree of molecular complexity seen in the $I S O$ results is well reproduced here.

Looking at the individual species in Table 3 , we see that our calculated column densities at a distance of $8.9 \times 10^{15} \mathrm{~cm}$ are within an order of magnitude of observed values for $\mathrm{CO}$, $\mathrm{CN}, \mathrm{NH}_{3}, \mathrm{CH}_{4}, \mathrm{C}_{2} \mathrm{H}, \mathrm{C}_{2} \mathrm{H}_{2}, \mathrm{C}_{6} \mathrm{H}_{2}$ and $\mathrm{C}_{6} \mathrm{H}_{6}$. Our calculated 
Table 3. Calculated and observed column densities $N\left(\mathrm{~cm}^{-2}\right)$ in CRL 618. Subscripts $\mathrm{C}$ and $\mathrm{P}$ refer, respectively, to calculated values at the chosen distance and peak values. The notation $x(y)$ represents $x \times 10^{y}$.

\begin{tabular}{lccclccc}
\hline \hline Species & $N_{\mathrm{C}}$ & $N_{\mathrm{P}}$ & \multicolumn{1}{c}{$N_{\mathrm{OBS}}$} & $\mathrm{Species}$ & \multicolumn{1}{c}{$N_{\mathrm{C}}$} & \multicolumn{1}{c}{$N_{\mathrm{P}}$} & $N_{\text {OBS }}$ \\
\hline $\mathrm{CN}$ & $1.8(14)$ & $5.5(16)$ & $6.5(14)^{a}$ & $\mathrm{HCN}$ & $1.7(15)$ & $9.0(17)$ & $5.8(14)^{b}-2.0(18)^{c}$ \\
$\mathrm{HNC}$ & $7.4(15)$ & $1.1(17)$ & $4.2(13)^{a}-1.0(16)^{d}$ & $\mathrm{NH}_{3}$ & $8.7(15)$ & $2.2(17)$ & $3.5(16)^{e, f}$ \\
$\mathrm{C}_{3}$ & $1.9(15)$ & & & $\mathrm{C}_{4}$ & $2.2(14)$ & & \\
$\mathrm{C}_{5}$ & $1.9(14)$ & & & $\mathrm{C}_{6}$ & $3.4(13)$ & & \\
$\mathrm{CH}_{4}$ & $1.7(16)$ & $2.5(17)$ & $7.0(16)^{e}$ & $\mathrm{C}_{2} \mathrm{H}_{4}$ & $1.8(13)$ & $4.8(14)$ & $5.0(16)^{e}$ \\
$\mathrm{C}_{2} \mathrm{H}$ & $2.1(14)$ & $2.4(14)$ & $2.0(15)^{b}$ & $\mathrm{C}_{3} \mathrm{H}$ & $2.8(15)$ & & \\
$\mathrm{C}_{4} \mathrm{H}$ & $4.2(13)$ & $3.7(13)$ & $1.2(15)^{b}$ & $\mathrm{C}_{5} \mathrm{H}$ & $2.0(15)$ & & \\
$\mathrm{C}_{6} \mathrm{H}$ & $2.5(15)$ & & & $\mathrm{C}_{7} \mathrm{H}$ & $3.6(14)$ & & \\
$\mathrm{C}_{8} \mathrm{H}$ & $6.7(14)$ & & & $\mathrm{C}_{2} \mathrm{H}_{2}$ & $4.4(16)$ & $5.7(18)$ & $2.0(17)^{g}$ \\
$\mathrm{C}_{3} \mathrm{H}_{2}$ & $9.2(13)$ & & & $\mathrm{C}_{4} \mathrm{H}_{2}$ & $6.3(15)$ & $2.3(17)$ & $1.2(17)^{g}$ \\
$\mathrm{C}_{5} \mathrm{H}_{2}$ & $1.9(15)$ & & & $\mathrm{C}_{6} \mathrm{H}_{2}$ & $1.4(16)$ & $7.7(16)$ & $6.0(16)^{g}$ \\
$\mathrm{C}_{7} \mathrm{H}_{2}$ & $7.5(15)$ & & & $\mathrm{C}_{8} \mathrm{H}_{2}$ & $7.6(15)$ & & \\
$\mathrm{C}_{3} \mathrm{~N}$ & $1.9(15)$ & & & $\mathrm{C}_{5} \mathrm{~N}$ & $4.0(14)$ & & \\
$\mathrm{HC}_{3} \mathrm{~N}$ & $1.7(16)$ & $6.0(16)$ & $1.3(14)^{a}-5.0(16)^{g}$ & $\mathrm{HC}_{5} \mathrm{~N}$ & $1.7(16)$ & $5.3(16)$ & $6.1(13)^{b}-1.5(16)^{g}$ \\
$\mathrm{HC}_{7} \mathrm{~N}$ & $2.2(16)$ & $2.5(16)$ & $<8.9(12)^{b}$ & $\mathrm{HC}_{9} \mathrm{~N}$ & $1.4(16)$ & & \\
$\mathrm{HC}_{11} \mathrm{~N}$ & $8.2(15)$ & & & $\mathrm{CH}_{3} \mathrm{C}_{2} \mathrm{H}$ & $2.4(14)$ & $2.4(15)$ & $1.8(16)^{e}$ \\
$\mathrm{CH}_{3} \mathrm{C}_{4} \mathrm{H}$ & $5.8(14)$ & $1.7(15)$ & $8.0(15)^{e}$ & $\mathrm{C}_{6} \mathrm{H}_{5} \mathrm{CN}$ & $1.7(15)$ & & \\
$\mathrm{C}_{6} \mathrm{H}_{6}$ & $9.0(15)$ & $4.5(16)$ & $5.0(15)^{g}$ & $\mathrm{H}_{2} \mathrm{CS}$ & $3.2(15)$ & & \\
$\mathrm{CS}^{9}$ & $9.0(15)$ & $4.2(17)$ & $1.9(13)^{a}$ & $\mathrm{C}_{2} \mathrm{~S}$ & $2.4(16)$ & & \\
$\mathrm{C}_{3} \mathrm{~S}$ & $4.9(15)$ & & & $\mathrm{C}_{4} \mathrm{~S}$ & $3.6(14)$ & & \\
$\mathrm{OCSS}$ & $2.5(14)$ & & & $\mathrm{CO}_{3}$ & $7.1(18)$ & $7.7(19)$ & \\
$\mathrm{H}_{3}^{+}$ & $4.3(10)$ & & & $\mathrm{HCO}^{+}$ & $3.1(11)$ & $1.7(12)$ & $3.2(13)^{a}$ \\
\hline
\end{tabular}

${ }^{a}$ Bachiller et al. (1997); ${ }^{b}$ Fukasaku et al. (1994); ${ }^{c}$ Thorwirth et al. (2003); ${ }^{d}$ Herpin \& Cernicharo (2000); ${ }^{e}$ Cernicharo et al. (2001a); ${ }^{f}$ Martín-Pintado et al. (1993); ${ }^{g}$ Cernicharo et al. (2001b) ${ }^{h}$ Justannont et al. (2000).

Table 4. Comparison of calculated, peak and observed fractional abundances for CRL 618, with respect to $n\left(\mathrm{H}_{2}\right)$. The calculated values are taken at a radial distance of $8.9 \times 10^{15} \mathrm{~cm}$. The notation $x(y)$ represents $x \times 10^{y}$.

\begin{tabular}{|c|c|c|c|c|c|c|}
\hline & $\mathrm{CO}$ & $\mathrm{CS}$ & $\mathrm{C}_{2} \mathrm{H}$ & $\mathrm{C}_{3} \mathrm{H}$ & $\mathrm{C}_{3} \mathrm{H}_{2}$ & $\mathrm{C}_{4} \mathrm{H}$ \\
\hline $8.9 \times 10^{15} \mathrm{~cm}$ & $6.0(-4)$ & $7.6(-7)$ & $1.8(-8)$ & $2.3(-7)$ & $7.8(-9)$ & $3.5(-9)$ \\
\hline Peak & $6.0(-4)$ & $4.0(-6)$ & $2.0(-8)$ & $7.7(-7)$ & $8.2(-9)$ & $5.0(-9)$ \\
\hline \multirow[t]{3}{*}{ Observed } & $8.0(-4)^{b}$ & $6.0(-8)^{a}$ & $2.0(-6)^{a}$ & $4.0(-8)^{a}$ & $2.0(-6)^{a}$ & $8.0(-8)^{a}$ \\
\hline & & $4.1(-7)^{b}$ & $2.0(-6)^{c}$ & & & \\
\hline & $\mathrm{HCO}^{+}$ & $\mathrm{HCN}$ & $\mathrm{HC}_{3} \mathrm{~N}$ & $\mathrm{HC}_{5} \mathrm{~N}$ & $\mathrm{HNC}$ & OCS \\
\hline $8.9 \times 10^{15} \mathrm{~cm}$ & $2.6(-11)$ & $1.4(-7)$ & $1.4(-6)$ & $1.5(-6)$ & $6.2(-7)$ & $2.1(-8)$ \\
\hline Peak & $5.9(-11)$ & $8.0(-6)$ & $1.6(-6)$ & $1.7(-6)$ & $1.6(-6)$ & $2.0(-7)$ \\
\hline \multirow[t]{3}{*}{ Observed } & $2.0(-7)^{a}$ & $5.0(-7)^{a}$ & $2.0(-7)^{a}$ & $1.0(-7)^{a}$ & $1.9(-6)^{b}$ & $<5.0(-7)^{a}$ \\
\hline & & $4.4(-6)^{b}$ & $2.9(-6)^{b}$ & & & \\
\hline & & $1.6(-7)^{c}$ & & & & \\
\hline
\end{tabular}

${ }^{a}$ Bujarrabal et al. (1988); ${ }^{b}$ Bujarrabal et al. (1994); ${ }^{c}$ Fuente et al. (1998).

results for $\mathrm{HC}_{3} \mathrm{~N}$ and $\mathrm{HC}_{5} \mathrm{~N}$ lie reasonably close to the upper range of the observed values. Both $\mathrm{HCN}$ and $\mathrm{HNC}$ are within the range of observed column densities given, and fractional abundances of these species agree well with observations. We appear to have too much $\mathrm{CS}$ and far too little $\mathrm{C}_{2} \mathrm{H}_{4}, \mathrm{C}_{4} \mathrm{H}, \mathrm{C}_{4} \mathrm{H}_{2}$, $\mathrm{CH}_{3} \mathrm{C}_{2} \mathrm{H}, \mathrm{CH}_{3} \mathrm{C}_{4} \mathrm{H}$ and $\mathrm{HCO}^{+}$. The larger molecule $\mathrm{HC}_{7} \mathrm{~N}$ is also very over-abundant relative to the observed column density (Fukasaku et al. 1994) but we note that this is derived from single-dish observations and that the $\mathrm{HC}_{5} \mathrm{~N}$ column density derived by them is a factor of 250 below the IR column density derived from the ISO observations. In summary, there is good agreement with $60 \%$ of observed species at the radius of 
$8.9 \times 10^{15} \mathrm{~cm}$, and moreover we are within an order of magnitude of observed column densities of all species, save $\mathrm{HCO}^{+}$, $\mathrm{C}_{2} \mathrm{H}_{4}$ and $\mathrm{C}_{4} \mathrm{H}$, at some radial point. A comparison of column densities is also shown in Fig. 3.

Since some observational data are presented as fractional abundances rather than column densities, we present in Table 4 a comparison between these observed values and the fractional abundances in our model at a radial distance of $8.9 \times 10^{15} \mathrm{~cm}$ and we also give peak abundances. By comparing results in this table with column densities in the previous table, one can yet again get a sense of the large uncertainty in observed values. For example, the ratio between the calculated and observed column densities for the ion $\mathrm{HCO}^{+}$is $1 \times 10^{-2}$ while the corresponding ratio for fractional abundances is $1.3 \times 10^{-4}$ ! Clearly, all that can be stated is that we underproduce $\mathrm{HCO}^{+}$in both cases, although the peak column density of $\mathrm{HCO}^{+}$is only a factor of twenty less than the observed value. To produce a much higher abundance in a dense warm gas would require such a large ionisation rate that the molecular gas would be destroyed rapidly (see Sect. 4.6 and Fig. 2). It may be that most of the $\mathrm{HCO}^{+}$resides in a different region from that which we are describing here, or as examined by Viti et al. (2002), the high $\mathrm{HCO}^{+}$levels might be due to some wind-interaction effects which we do not address: Rawlings et al. (2000), in a paper which contributes results to Viti et al., show that the interaction between slow moving $\left(\sim 1 \mathrm{~km} \mathrm{~s}^{-1}\right)$ gas and a fast $\left(\sim 100 \mathrm{~km} \mathrm{~s}^{-1}\right)$ outflow can enhance the chemistry of $\mathrm{HCO}^{+}$. Viti et al. show that this enhancement can mean higher abundances by an order of magnitude. The formation of $\mathrm{HCO}^{+}$in this case requires the presence of $\mathrm{H}_{2} \mathrm{O}$, which although not included in our model, is present in CRL 618 (Herpin \& Cernicharo 2000). Despite observational uncertainties as well as the problem that some species have rapidly changing abundances in the neighbourhood of the photodissociation distance, the general agreement is encouraging, particularly for the cyanopolyynes, $\mathrm{HCN}$, and HNC. On the other hand, the calculated fractional abundances of some hydrocarbons tend to be too low.

The model results reported here are for an isothermal system at a temperature of $250 \mathrm{~K}$. We have also considered two other models which start at temperatures of $250 \mathrm{~K}$ and $800 \mathrm{~K}$, respectively, and in which the temperature decreases as the slab moves outward. Both models can be justified partially by observations: according to Herpin \& Cernicharo (2000), the torus of CRL618 lies at $800 \mathrm{~K}$ but most of the molecular emission comes from the $250 \mathrm{~K}$ region. These models show that the effects of a radial temperature dependence on abundances are negligible. Note that the network of rate coefficients may not be entirely accurate at temperatures as high as $800 \mathrm{~K}$.

\section{Conclusions}

We have shown that a model of a dense, warm gaseous slab, irradiated by an intense UV field, is able to generate very large abundances of large, complex molecules, including benzene. Assuming a dense slab/torus expanding at $5 \mathrm{~km} \mathrm{~s}^{-1}$, the phase during which molecules such as benzene are produced lasts 500 years, which is similar to the predicted age of CRL 618. The derived column densities give a reasonable agreement with the ISO and millimetre-wave observations of organic molecules in CRL 618, although it is often difficult to reconcile radio and IR observations of the same molecules. Our physical model of a slowly expanding and uniform slab simplifies the true complexity of the source; different results would be obtained with more complex physical morphologies. Future work should take into account the complex physical structure of CRL 618.

Acknowledgements. We are grateful to ESO for support of PMW through a studentship. Astrophysics at UMIST is supported by a grant from PPARC. The astrochemistry program at The Ohio State University is supported by the National Science Foundation.

\section{References}

Ali, A., Shalabiea, O. M., El-Nawawy, M. S., \& Millar, T. J. 2001, MNRAS, 325, 881

Bachiller, R., Forveille, T., Huggins, P. J., \& Cox, P. 1997, A\&A, 324, 1123

Bujarrabal, V., Gómez, J., Bachiller, R., \& Martín-Pintado, J. 1988, A\&A, 204, 242

Bujarrabal, V., Fuente, A., \& Omont, A. 1994, A\&A, 285, 247

Bujarrabal, V., Castro-Carrizo, A., Alcolea, J., \& Sánchez Contreras, C. 2001, A\&A, 377,868

Carsenty, U., \& Solf, J. 1982, A\&A, 106, 307

Cernicharo, J. 2000, in Astrochemistry: From Molecular Clouds to Planetary Systems, ed. Y. C. Minh, \& E. F. van Dishoeck, ASP, IAU Symp., 197, 375

Cernicharo, J., Guélin, M., Martín-Pintado, J., Peñalver, J., \& Mauersberger, R. 1989, A\&A, 222, L1

Cernicharo, J., Heras, A. M., Pardo, J. R., et al. 2001a, ApJ, 546, L127

Cernicharo, J., Heras, A. M., Tielens, A. G. G. M., et al. 2001b, ApJ, 546, L123

Fong, D., Meixner, M., Castro-Carrizo, A., et al. 2001, A\&A, 367, 652

Fuente, A., Cernicharo, J., \& Omont, A. 1998, A\&A, 330, 232

Fukasaku, S., Hirahara, Y., Masuda, A., et al. 1994, ApJ, 437, 410

Fukuzawa, K., \& Osamura, Y. 1997, ApJ, 489, 113

Glassgold, A. E., Najita, J., \& Igea, J. 1997, ApJ, 480, 344

Guerrero, M. A., Chu, Y.-H., \& Gruendl, R. A. 2000, ApJS, 129, 295

Hajian, A. R., Phillips, J. A., \& Terzian, Y. 1996, ApJ, 467, 341

Hasegawa, T., Volk, K., \& Kwok, S. 2000, ApJ, 532, 994

Herpin, F., \& Cernicharo, J. 2000, ApJ, 530, L129

Hoobler, R. J., \& Leone, S. R. 1997, J. Geophys. Res., 102, 28717

Howe, D. A., \& Millar, T. J. 1990, MNRAS, 244, 444

Howe, D. A., Millar, T. J., \& Williams, D. A. 1992, MNRAS, 255, 217

Howe, D. A., Hartquist, T. W., \& Williams, D. A. 1994, MNRAS, 271, 811

Hunter, S. D., Bertsch, D. L., Catelli, J. R., et al. 1997, ApJ, 481, 205

Jura, M., Balm, S. P., \& Kahane, C. 1995, ApJ, 453, 721

Justtanont, K., Barlow, M. J., et al. 2000, A\&A, 360, 1117

Knapp, G. R., Crosas, M., Young, K., \& Ivezic, Z. 2000, ApJ, 534, 324

Lo, K. Y., \& Bechis, K. P. 1976, ApJ, 205, L21

Mamon, G. A., Glassold, A. E., \& Huggins, P. J. 1988, ApJ, 328, 797

McEwan, M. J., Scott, G. B. I., Adams, N. G., et al. 1999, ApJ, 513, 287

Martín-Pintado, J., Gaume, R. A., Bachiller, R., \& Johnston, K. J. 1993, ApJ, 419, 725

Martín-Pintado, J., Gaume, R. A., Johnston, K. J., \& Bachiller, R. 1995, ApJ, 446, 687

Matsuura, M., Zijlstra, A. A., van Loon, J. Th., et al. 2002, ApJ, 580, L133 
Meixner, M., Campbell, M. T., Welch, W. J., \& Likkel, L. 1998, ApJ, 509,392

Melnick, G. J., Neufeld, D. A., Ford, K. E., et al. 2001, Nature, 412, 160

Millar, T. J. 1988, in Rate Coefficients in Astrochemistry, ed. T. J. Millar, \& D. A. Williams (Dordrecht: Kluwer Academic Publishers), 287

Millar, T. J., \& Herbst, E. 1994, A\&A, 288, 561

Millar, T. J., Herbst, E., \& Bettens, R. P. A. 2000, MNRAS, 316, 195

Millar, T. J., Flores, J. R., \& Markwick, A. J. 2001, MNRAS, 327, 1173

Neri, R, Kahane, C., Lucas, R., Bujarrabal, V., \& Loup, C. 1998, Ap\&SS, 130, 1

Neri, R., García-Burillo, S., Guélin, M., et al. 1992, A\&A, 262, 544

Olofsson, H. 1997, in Molecules in Astrophysics: Probes and Processes, ed. E. F. van Dishoeck (Dordrecht: Kluwer), 457

Olofsson, H., \& Nyman, L.-Å. 1999, A\&A, 347, 194
Phillips, J. P., Williams, P. G., Mampaso, A., \& Ukita, N. 1992, A\&A, 260, 283

Rawlings, J. M. C., Taylor, S. D., \& Williams, D. A. 2000, MNRAS, 313,461

Soker, N., \& Kastner, J. H. 2002, ApJ, 570, 245

Sopka, R. J., Olofsson, H., Johansson, L. E. B., Nguyen-Q-Rieu, \& Zuckerman, B. 1989, A\&A, 210, 78

Speck, A. K., Meixner, M., \& Knapp, G. R. 2000, ApJ, 545, L145

Strong, A. W., \& Moskalenko, I. V. 1998, ApJ, 509, 212

Thorwirth, S., Wyrowski, F., Schilke, P., et al. 2003, ApJ, 586, 338

Truong-Bach, Graham, D., \& Nguyen-Q-Rieu 1993, A\&A, 277, 133

Viti, S., Natarajan, S., \& Williams, D. A. 2002, MNRAS, 336, 797

Woods, P. M., Millar, T. J., Zijlstra, A. A., \& Herbst, E. 2002, ApJ, 574, L167

Zijlstra, A. A., Chapman, J. M., te Lintel Hekkert, P., et al. 2001, MNRAS, 322, 280 